\title{
The Relationship between Creative Inspiration, Self-Evaluated Satisfaction, and Brain Activity During Simplified Music Composition
}

\author{
Hiromu SATO* Non-member, \\ KENJI MORIYA $^{\ddagger \S}$ Member, \\ YUYA CHIBA $^{\dagger}$ \\ Non-member \\ MASAHIRO NAKAGAWA ${ }^{\mathbb{I}} \quad$ Non-member
}

(Received April 8, 2021, revised July 13, 2021)

\begin{abstract}
The human prefrontal cortex (PFC) is an important target for research that attempts to elucidate and influence higher brain function using non-invasive neuroimaging techniques. In particular, creativity is a specific higher human brain function and is integral for the evolution of human society. Creative inspirations generated during creative activity often provide solutions to hard problems that lead to a progressive society. The present study examined PFC activity when creative inspiration occurred and evaluated the influence of brain activity on the quality of creation using the simplified music composition task and near-infrared spectroscopy (NIRS). Deeply understanding these brain functions possibly builds a foundation for the development of methods to stimulate brain activation for the simulation of creativity. The PFC activity measurements were achieved by measuring oxygen metabolism in cerebral blood flow (CBF) using wearable ten-channel optical topography. We invented a new, simplified method of music composition to analyze brain activity of a person without musical instrument experience. A report of creative inspirations was conducted by asking the subject to raise their hand to self-report instances of inspiration during the music composition task that were then compared against statistically analyzed brain activity before and after self-reported inspirations. A correlation coefficient between brain activity and self-evaluation measured by the visual analog scale (VAS) was investigated. The present research found that the right ventrolateral PFC is activated by creative inspiration. Significant brain activation of four out of five participating subjects was observed $(p<0.05)$. CBF increases delayed from inspirations, and mean delay time was approximately $12 \mathrm{~s}$. We observed weak positive correlation between brain activity and self-evaluation in a wide range. However, the correlation coefficient was not statistically significant $(p>0.05)$. The simplicity of the music composition task possibly masks clear correlation. In conclusion, the main finding is that right PFC activation via self-reported inspiration indicates an important mechanism of creativity.
\end{abstract}

Keywords: Near-infrared spectroscopy, Brain activity, Creative inspiration, Music composition,

\section{Introduction}

To bring out and freely control brain ability is a desirable goal for the improvement of an enriching lifestyle. Nevertheless, human brain mechanisms, especially those involving creativity, complex emotions, and self-consciousness, are not sufficiently delineated. The elucidation of these mechanisms is helpful and important not only for the improvement and individual quality of life but also for the application in various commercial and medical fields. The

\footnotetext{
¥ Corresponding: moriya@hakodate-ct.ac.jp

* Advanced Course of Production Systems Engineering, National Institute of Technology, Hakodate College (Graduate)

Tokura-cho 14-1, Hakodate City, Hokkaido, Japan 042-8501

$\dagger$ Support Center for Engineering Education, National Institute of Technology, Hakodate College

Tokura-cho 14-1, Hakodate City, Hokkaido, Japan 042-8501

$\S$ Department of Production Systems Engineering, National Institute of Technology, Hakodate College

Tokura-cho 14-1, Hakodate City, Hokkaido, Japan 042-8501

II Department of Electrical, Electronics and Information Engineering, Nagaoka University of Technology

1603-1, Kami-Tomioka-cho, Nagaoka City, Niigata, Japan 940-2137
}

prefrontal region of the human brain is a focus of the field of higher brain functional research since this region is developing in contrast to other animals. In order to systematically understand this quality of the prefrontal cortex (PFC), research has examined emotion estimation using brain activity in a wide range from children to adults relative to higher brain functions [1] [3].

Alternately, creativity is also an important brain function for the improvement of technology, culture, and individual lifestyles. Actually, creative activities and artistic creations such as music are also important factors in healing and relaxation during daily life [4]. When we engage in these creative activities, we often receive creative inspiration that results in good ideas suddenly coming to mind. Inspiration can cause people to obtain high satisfaction since it can create outstanding objects, solve serious problems, and lead circumstances in a good direction. Previous inspiration studies have demonstrated that the cerebral blood flow (CBF) in the PFC increases due to inspiration that is experienced during morphing image change detection tasks [5]. In addition, previous studies that use creative tasks in- 
dicated that bilateral frontal activity in musicians is greater than in non-musicians during creative tasks and that creativity is supported by increased frontal cortical activity [6]. However, research that focuses on creative inspiration has attempted to reveal that the relationship between creative inspiration and brain activity has not adequately explained [7]. Creativity is based on divergent thinking that seeks ideas in various fields without the restriction of boundaries and creates ideas including flexible solutions to open-ended problems [8]. Therefore, we guess that using music is suitable for examining creative inspiration due to the ease of searching for ideas and receiving inspirations during music-related activities involving abundant experiences and knowledge accumulated inside of people. The present research focused on creative activity in which the formation of good ideas and enthusiasm occasionally enables performing high-quality work during a project. We adopted music composition as a creative task and invented a new, simplified music composition method that allows for simplified music production without prior education.

Neuroimaging techniques such as electroencephalography (EEG) and functional magnetic resonance imaging (fMRI) are frequently used for the measurement of human brain activity since both methods are non-invasive. Similarly, in recent years, neuroscientists frequently used near infrared spectroscopy (NIRS) as an index of brain activity, despite the technique having several drawbacks in comparison to other neuroimaging techniques. For instance, NIRS can only obtain information about the brain's surface layer and has poor spatial resolution. Nevertheless, NIRS presents several distinguished advantages compared with other measuring methods: simplicity of use and administration, less physical and psychological burden compared to existing neuroimaging techniques due to low restriction of body movement, and high robustness with extrinsic noise in the environment such as electromagnetic waves [9]. Thus, NIRS can measure brain activity in a state that can be obtained in ordinary life and is suitable for experiments using sound elements such as music because extrinsic noise can be avoided.

The PFC supports higher brain functions such as creativity. Additionally, heuristic decision-making is also supported by the PFC and is associated with the activation of the orbitofrontal cortex (OFC) [10]. Thus, we speculated that creativity or creative inspiration is related to OFC activity and hypothesized that the ventral PFC is activated by creative inspiration. We measured characteristics of brain activity change when creative inspiration was reported and investigated the relationship between self-evaluation and brain activity during a music composition task. Investigating this may provide a foundation for the elucidation of the relationship between creativity and brain activity and the development of methods to stimulate brain activation for the simulation of creativity by incorporating brain stimulation methods that are investigated actively and applied clinically [11][12].

\section{Experimental Method}

2.1 Participants We examined data from healthy five participants (five males, age range: 19-21 years) who are affiliated with the National Institute of Technology, Hakodate College, as students. In accordance with the regulation set forth by the Life Ethics Committee of the National Institute of Technology, Hakodate College, we explained details and the safety of the experiment, and informed consent was obtained from all participants. Only one participant out of five participants had experience playing the keyboard instrument, but the other four participants did not have experience with musical instruments. All participants have never composed music but have listened to music on a near-daily basis.

2.2 Creative Task: Simplified Music Composition [13] The music composition task involved the composition of instrumental pieces of rock music chosen considering the amount of musical knowledge and experience of the subjects. We created three instrument phrases in a common key (A-minor) and tempo (120 bpm) in advance: drum phrases that were prepared 11 phrases each length of eight bars via programing; accompaniment electric guitar phrases that were prepared with seven kinds of chord progression each length of eight bars based on diatonic chords by recording electric guitar phrases. Moreover, these seven chord progressions were created in three sets and were labeled "slow," "middle," and "fast" by the amount of sounds per bar; melodious electric guitar phrases that were prepared 11 phrases each length of four bars to create various combinations in the same manner as the accompaniment electric guitar. These phrases were composed of the A-minor pentatonic scale (notes: A, C, D, E, G). Melodious electric guitar phrases were also labeled "slow," "middle," and "fast," similar to the accompaniment electric guitar, to roughly indicate phrases amenable to the subjects. We asked each subject to compose music by choosing and combining these phrases freely using digital audio workstation (DAW) software on a personal computer without playing the instruments. The entire length of each sample of created music was 24 bars and was separated into three sections, each containing eight bars. The first section is created in advance for simplicity. Thus, the subjects created two sections of the first section continuous. Figure 1 illustrates the overview of the music composition methods.

2.3 Creative Inspiration In the present study, we defined creative inspiration as the following: subjects come up with a good plan, idea of composition, and phrase without being prompted; subjects receive some stimulus from prepared musical phrases or created pieces. We explained these definitions to all subjects before the beginning of experiments. Subjects reported inspiration by judging subjective perceptions according to these definitions.

2.4 Experimental Procedure We quantitatively evaluated the brain activity of each subject as they performed a music composition task following a rest task. During the experiment, the subject sat on a chair facing the screen of a personal computer. The subject was instructed to keep their 


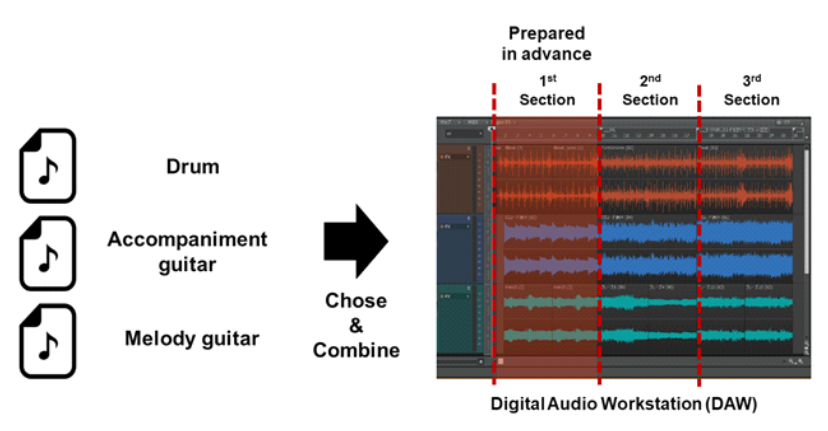

Figure 1: Illustration of the simplified music composition method. Subjects choose prepared phrases and combine them on the DAW software. The light fill part on the DAW picture indicates the first section that was created in advance.

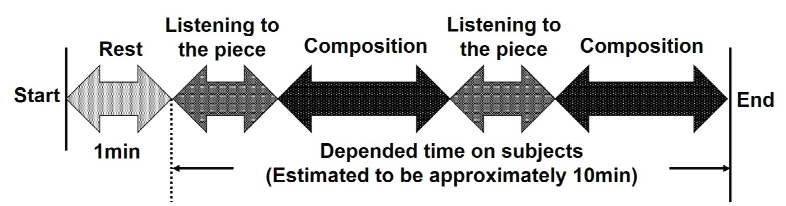

Figure 2: Timeline illustrating the experimental procedure.

eyes closed during the rest task. The experimental procedure is shown in Fig. 2. The first minute (min) of the procedure comprised a set "rest" period. During the "listening to the piece" period, we asked the subjects to listen to the first section of a piece of music until memorization. After these tasks, the subjects proceeded to the "composition" task. The cycle of the "composition" and "listening to the piece" tasks was repeated two times each experiment since pieces that should be created by the subjects were separated into two sections. In the second "listening to the piece," the subjects listened to the first and second section pieces of music until the subjects memorize. When the subjects received an inspiration, we asked the subjects to raise their hands as possible as small, and we recorded the time. After they composed two sections, the subjects evaluated selfevaluation of the created respective piece subjectively using the visual analog scale (VAS). VAS is a frequently used method to assess subjective sensibility and feeling in various fields including medical field. It consists of a line usually $100 \mathrm{~mm}$ in length, with index description such as "not satisfy" and "satisfy significantly" in both ends of a line. The subjects reflected their perception and marked point on a line. We measured the location of points to $1[\mathrm{~mm}] \mathrm{accu}-$ racy. Thus, these results became 101-point scale from 0 to 100 points [14] [15].

2.5 Measurement and Analysis of Brain Activity We quantitatively evaluated brain activity in the PFC by measuring changes in the concentration of oxygenated and deoxygenated hemoglobin (oxy-Hb and deoxy-Hb, respectively) using ten-channel wearable optical topography (WOT-100, NeU Ltd.) with NIRS at a sampling frequency of $5 \mathrm{~Hz}$. This method uses near-infrared ray $(700-900 \mathrm{~nm})$

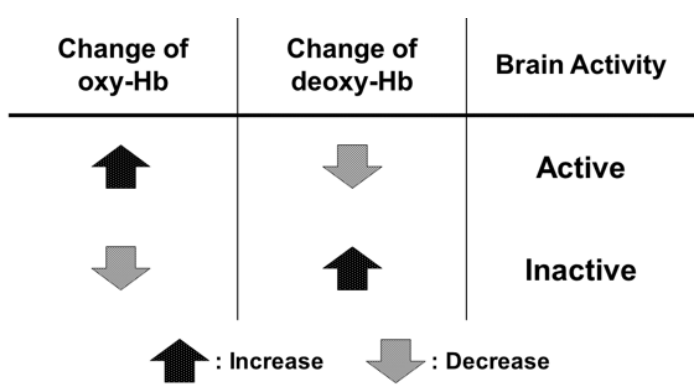

Figure 3: Relationship of brain activity with changes in concentrations of oxygenated and deoxygenated hemoglobin.

that has high bio-permeability. With the change in the oxidation state of hemoglobin, the intensity of the transmitted near-infrared ray changes because the absorption spectra of oxy-Hb and deoxy-Hb are different. Therefore, the change in oxidation state can be evaluated from the intensity change of the ray. These indexes are highly associated with brain functional activation, thus providing reliable indicators of nervous activity for several different tasks. CBF increases during neural activity are greater than associated activity increases of oxygen metabolism such as oxygen consumption in neuron [16] [17]. This imbalance between supply and demand that is called neurovascular uncoupling indicates that oxy-Hb follows a $\mathrm{CBF}$ change and decrease in deoxy- $\mathrm{Hb}$ as a result of neural activity. Therefore, typically, an increase in oxy-Hb and a decrease in deoxy-Hb indicate that the PFC is active compared with the previous condition, and a decrease in oxy-Hb and an increase in deoxy-Hb indicate that the PFC is inactive (Fig. 3).

We used the 15-s mean value to analysis considering the time lag between neuronal response and a CBF change, rising and falling time [18]. To eliminate the influence of sudden change, $15 \mathrm{~s}$ of before switch between task and after one was excluded from analysis. The duration of music composition task was separated into 15-s segments, and the mean data was used in analysis. The measurement regions of the PFC corresponding to each measurement channel $(\mathrm{CH})$ are shown in Fig. 4. We investigated the following two factors: to evaluate the effect of creative inspiration to brain functional activation, we calculated mean values of oxy- and deoxy-Hb concentration change in $15 \mathrm{~s}$ after inspirations were reported and before in $15 \mathrm{~s}$ and conducted a statistical test whether there is a significant difference at a significance level of $5 \%$; to confirm correlation between brain functional activation and subjective self-evaluation, we investigated the relationship between average values of oxy- $\mathrm{Hb}$ density change when brain activity was activated and selfevaluation that was measured using VAS. Similarly, correlation coefficient was verified statistically at a significance level of $5 \%$.

\section{Results and Discussion}

We conducted the experiment each three times to all subjects, thus obtaining 30 data of brain activity during the music composition task. In total, creative inspirations were reported 15 times: subject A (a person with keyboard in- 


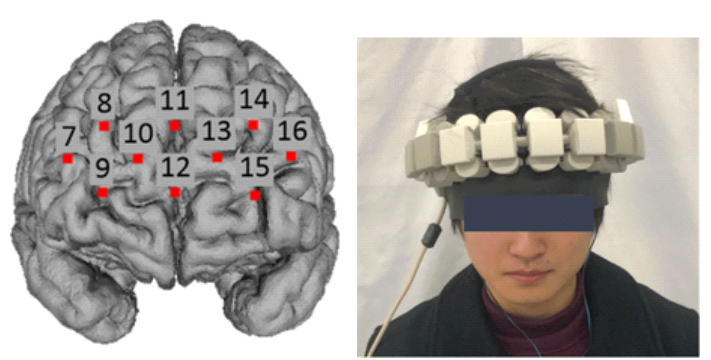

Figure 4: Regions of the prefrontal cortex (left image) corresponding to the measurement channels of the present study. Measurements of oxygenated and deoxygenated hemoglobin concentrations were conducted using tenchannel wearable optical topography (right image).

* Brain image (left image) is reused from reference [1].

strument experience) reported eight times; subject B reported one time; subject $\mathrm{C}$ reported two times; subject $\mathrm{D}$ reported three times; subject E reported one time. Subject A reported many inspirations compared with other subjects. This amount difference of inspirations implied the difference between a person who has music experience and a person who does not have.

3.1 Brain Activity Related to Creative Inspiration In several experiments, a CBF change in all channels had a tendency to increase after creative inspirations were reported. In analysis through all experiments using paired ttest, we observed significant oxy-Hb increases and deoxy$\mathrm{Hb}$ decreases in $9 \mathrm{CH}$ only $(p<0.05)$, namely, right ventrolateral PFC was activated by creative inspiration. A CBF change was used to demonstrate oxy-Hb. Figure 5 illustrates 30-s mean oxy-Hb concentration change in $9 \mathrm{CH}$ after creative inspirations were reported using mean previous 15 $\mathrm{s}$ value as control. In Fig. 5, each oxy-Hb change of the subjects was standardized using maximum value of each subject. We deducted the data of subject $\mathrm{C}$ from this analysis since oxy-deoxy-Hb density change both decreased; we could not judge active or inactive following the definition of Fig. 3. Regarding a CBF change, subject A did not have unique reaction in comparison to other subjects unlike the amount of creative inspiration. A speed fluctuation of a CBF had difference in every subject. In particular, fluctuation of subject B was fast; on the contrary, fluctuation of subject D was slow. A peak of every subject's $\mathrm{CBF}$ increases delayed from creative inspiration, and average was approximately $12 \mathrm{~s}$. Inspiration intensity possibly influenced this difference of fluctuation.

The data from this experiment supported our hypothesis that ventral PFC is activated by creative inspiration. Moreover, several previous researches reported that creativity was associated with the right PFC and also supports our result. An fMRI study of high-level cognitive constructs such as creativity found creativity is related to the ability of the right dorsolateral PFC and sensorimotor areas [19]. During a divergent thinking task that creates stories, the right PFC was activated significantly [20]. There is a possibility that creative inspiration triggers this brain functional activation.

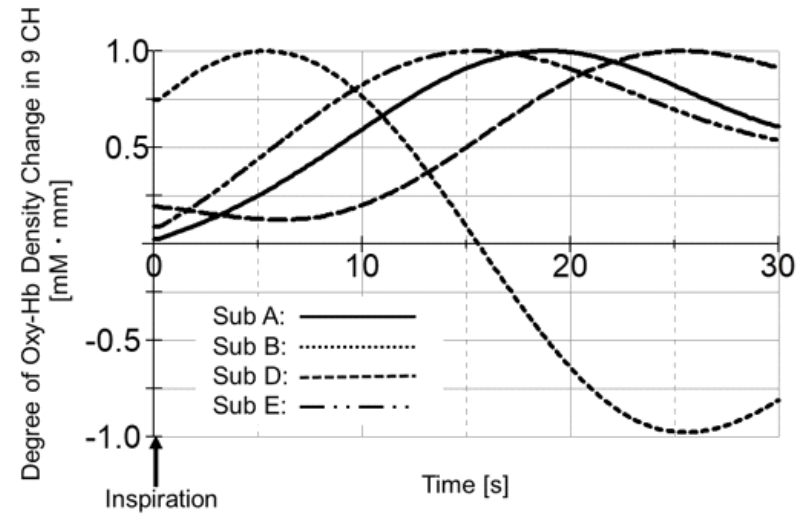

Figure 5: Mean oxy-Hb concentration change of each subject in $9 \mathrm{CH}$ for $30 \mathrm{~s}$ after creative inspiration. Point of zero second shows time during which creative inspirations were reported.

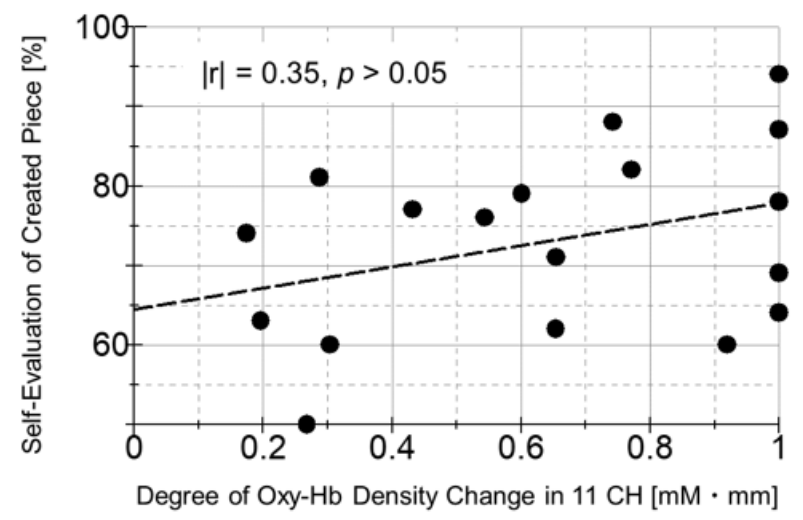

Figure 6: Correlation between mean oxy-Hb concentration change of all subjects in $11 \mathrm{CH}$ and self-evaluation.

\subsection{Relationship between self-evaluation and brain ac-} tivity Mean oxy-Hb concentration change, based on Fig. 3, when the PFC was activated during the music composition task was used to demonstrate brain activity using a mean oxy-Hb change during the rest task as a control. In particular, we focused on the medial PFC, namely, 11 and $12 \mathrm{CH}$, since previous research reported that pleasant and unpleasant emotions could be assessed using the medial PFC activity [21]. The correlation analysis between self-evaluation measured by VAS and brain activity indicated a positive correlation. Nevertheless, this correlation coefficient was not statically significant $(p>0.05)$. Figure 6 presents the correlation between self-evaluation and brain activity in $11 \mathrm{CH}$ that was standardized using the maximum value of each subject. Self-evaluation values had high scores in every subject $(M=72, S D=11, N=30)$. We considered that this high score tendency was attributed to the simplification of the music composition task and masked the correlation. Phrases that were prepared in advance were difficult to deviate from because they were composed based on several combinations of diatonic chords and pentatonic scales of the same key. In brief, there was the possibility that all subjects can easily create moderate quality creations. 
Elucidation of relationships between self-satisfaction and brain activity is essential for the development of brain activation methods to propose outstanding creativity. We have to adopt other indexes of self-satisfaction such as subjective progress or enjoyment instead of self-evaluation.

\section{Conclusion}

We could not analyze the network of each cortex, specifically due to the difficulty of measuring a wide cortex area, so we examined the brain activity related to creative inspiration and correlation between self-evaluation and brain activity in the PFC. The data of previous experiments supported our hypothesis of brain functional activation by creative inspiration. We did not obtain obvious correlation between self-evaluation and brain activity in the second experiment. An important finding in the present research was the activation of the $9 \mathrm{CH}$, namely, the right ventrolateral PFC, by creative inspiration. This result has similarities with previous research, which reported association of creativity and right $\mathrm{PFC}$ activation. In addition, intensity of creative inspiration possibly influences a $\mathrm{CBF}$ change since significant fluctuation occurred. Therefore, we should measure the intensity of inspirations subjectively using VAS in future work and verified influence.

The difference of inspiration amount between a person with experience of playing instruments and a person without experience of playing instruments was observed. The problems regarding the differences between experienced brain activity and the relationship between brain activity and amount of inspiration were generated. However, we could not create an argument using statistical terms because of the small sample size in this study. The goal of our research is the development of brain activation methods for creativity and providing good quality creative activity for artists, researchers, and developers. In order to achieve this goal, we should conduct further investigations that measure differences in brain activity between people experienced in playing musical instruments and musical composition and ordinary people and that use other indexes such as subjective progress or enjoyment as evaluations of creative activity with an increased number of subjects.

\section{Additional statement}

This research was approved as making adequate provisions for the safety and privacy of subjects by the Life Ethics Committee of National Institute of Technology, Hakodate College.

\section{Acknowledgment}

A part of this study was supported by a grant from Nagaoka University of Technology for collaborative research with National Institute of Technology 2019, 2020.

The authors would like to thank Enago (www.enago.jp) for the English language review.

\section{References}

[1] K. Moriya, I. Kurimoto, N. Ezaki and M. Nakagawa, "Influences of Listening to Music in Study Break on Brain Activity and Parasympathetic Nervous System Activity", Journal of the Institute of Industrial Applications Engineers, Vol.6, No.1, pp.34-38, 2018. DOI: 10.12792/jiiae.6.34

[2] M. Mauri, A. Crippa, A. Bacchetta, S. Grazioli, E. Rosi, E. Gazzola, A. Gallace and M. Nobile, "The utility of NIRS technology for exploring emotional processing in children", Journal of Affective Disorders, Vol.274, No.6, pp.819-824, 2020. DOI: 10.1016/j.jad.2020.06.004

[3] H. Doi, S. Nishitani and K. Shinohara, "NIRS as a tool for assaying emotional function in the prefrontal cortex", Frontiers in Human Neuroscience, Vol.7, No.770, pp.1-6, 2013. DOI: 10.3389/fnhum.2013.00770

[4] Y. Mitani, T. Kubo, Y. Chiba, Y. Maruyama, K. Moriya and M. Nakagawa, "Brain Activity During Listening to And Imagining Music: Does Imagining Music Provide a Similar Effect as Listening to Music?", Journal of the Institute of Industrial Applications Engineers, Vol.7, No.4, pp.127-131, 2019.

[5] K. Teranishi and H. Hagiwara, "Characteristic Changes in the Brain Measured by Near-Infrared Spectroscopy (NIRS) during "Aha” Experiences", J. Mobile Interactions, Vol.1, No.1, pp.41-46, 2011.

[6] C. Gibson, B. S. Folley and S. Park, "Enhanced divergent thinking and creativity in musicians: A behavioral and nearinfrared spectroscopy study", Brain and Cognition, Vol.69, No.1, pp.162-169, 2009. DOI: 10.1016/j.bandc.2008.07.009

[7] H. Sato, Y. Chiba, Y. Maruyama, K. Moriya and M. Nakagawa, "Investigation of Relationship Between Progress of Creative Activity and Brain Activity", Proceedings of the 8th IIAE International Conference on Industrial Application Engineering, pp.21-25, 2020. DOI: 10.12792/iciae2020.007

[8] S. A. Mednick, "The associative basis of the creative process", Psychological Review, Vol.69, No.3, pp.220-232, 1962. DOI: $10.1037 / \mathrm{h} 0048850$

[9] V. Quaresima, S. Bisconti and M. Ferrari, "A brief review on the use of functional near-infrared spectroscopy (fNIRS) for language imaging studies in human newborns and adults", Brain and Language, Vol.121, No.2, pp.79-89, 2012. DOI: 10.1016/j.bandl.2011.03.009

[10] P. H. Rudebeck and E. L. Rich, "Orbitofrontal Cortex", Current Biology, Vol.28, No.18, pp.R1083-R1088, 2018.

[11] U. R. Mohan, A. J. Watrous, J. F. Miller, B. C. Lega, M. R. Sperling, G. A. Worrell, R. E. Gross, K. A. Zaghloul, B. C. Jobst, K. A. Davis, S. A. Sheth, J. M. Stein, S. R. Das, R. Gorniak, P. A. Wanda, D. S. Rizzuto, M. J. Kahana and J. Jacobs, "The effects of direct brain stimulation in humans depend on frequency, amplitude, and white-matter proximity”, Brain Stimulation, Vol.13, No.5, pp.1183-1195, 2020. DOI: 10.1016/j.brs.2020.05.009 
[12] Y. Huang, M. Lu, A. Antal, J. Classen, M. Nitsche, U. Ziemann, M. Ridding, M. Hamada, Y. Ugawa, S. Jaberzadeh, A. Suppa, W. Paulus and J. Rothwell, "Plasticity induced by non-invasive transcranial brain stimulation: A position paper", Clinical Neurophysiology, Vol.128, No.11, pp.23182329, 2017. DOI: 10.1016/j.clinph.2017.09.007

[13] H. Sato, Y. Chiba, K. Moriya and M. Nakagawa, "Relationship between Creative Inspiration, Self-Evaluated Satisfaction and Brain Activity through Simplified Music Composition", Proceedings of the 9th IIAE International Conference on Industrial Application Engineering 2021, pp.123129, 2021.

[14] V. Ducoulombier, R. Chiquet, S. Graf, B. Leroy, G. Bouquet, S. Verdun, F. Martellier, A. Versavel, A. Kone, K.Lacroix, D. Duthoit, Q. Lenglet, A. Devaux, R. Jeanson, A. Lefebvre, B. Coviaux, G. Calais, A. Grimbert, M. Ledein, M. Moukagni, T. Pascart and E. Houvenagel, "Usefulness of a Visual Ana$\log$ Scale for Measuring Anxiety in Hospitalized Patients Experiencing Pain: A Multicenter Cross Sectional Study", Pain Management Nursing, Vol.21, No.6, pp.572-578, 2020. DOI: 10.1016/j.pmn.2020.03.004

[15] S. Watanabe and Y. Matsumoto, "An Analysis of Information Skills for Students Based on a Visual Analog Scale", Biomedical Fuzzy Systems Association, Vol.13, No.1, pp.5762, 2011 (in Japanese).

[16] D. Attwell, A. M. Buchan, S. Charpak, M. Lauritzen, B. A. MacVicar and E. A. Newman, "Glial and neuronal control of brain blood flow", Nature, Vol.468, No.7321, pp.232-243, 2010. DOI: $10.1038 /$ nature09613

[17] C. Leithner, G. Royl, N. Offenhauser, M. Füchtemeier, M. Kohl-Bareis, A. Villringer, U. Dirnagl and U. Lindauer, "Pharmacological uncoupling of activation induced increases in CBF and $\mathrm{CMRO}_{2}$ ", Journal of Cerebral Blood Flow \& Metabolism, Vol.30, No.2, pp.311-322, 2009. DOI: $10.1038 / \mathrm{jcbfm} .2009 .211$

[18] Y. Yang, W. Engelien, H. Pan, S. Xu, D. A. Silbersweig and E. Stern, "A CBF-Based Event-Related Brain Activation Paradigm: Characterization of Impulse-Response Function and Comparison to BOLD", NeuroImage, Vol.12, No.3, pp.287-297, 2000. DOI: 10.1006/nimg.2000.0625

[19] Y. N. Kenett, J. D. Medaglia, R. E. Beaty, Q. Chen, R. F. Betzel, S. L. Thompson-Schill and J. Qiu, "Driving the brain towards creativity and intelligence: A network control theory analysis", Neuropsychologia, Vol.118, No.12, pp.79-90, 2018. DOI: 10.1016/j.neuropsychologia.2018.01.001

[20] Paul A. Howard-Jones, Sarah-Jayne Blakemore, Elspeth A. Samuel, Ian R. Summers and Guy Claxton, "Semantic divergence and creative story generation: An fMRI investigation", Cognitive Brain Research, Vol.25, No.1, pp.240-250, 2005.

[21] Akinori Hirano, Shuichi Yamamoto, Kazuki Yanagisawa and Hitoshi Tsunashima, "Evaluation of pleasant and unpleasant emotion evoked by visual stimuli using NIRS", Journal of the Human Interface Society, Vol.17, No.3, pp.297-306, 2015 (in Japanese). DOI: 10.1109/ICCAS.2015.7364944

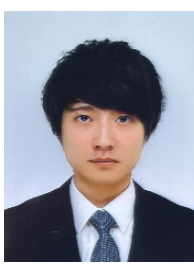

Hiromu Sato (Non-member) received Bachelor degree in engineering from National Institute of Technology (KOSEN), Hakodate College in 2021. He is presently student of Course of Bioengineering and Bioinformatics, Faculty of Information Science and Technology, Hokkaido University.

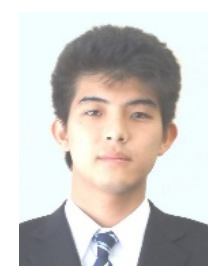

Yuya Chiba (Non-member) received Associated degree in engineering from National Institute of Technology (KOSEN), Hakodate College in 2012, and is presently technical officer of NIT (KOSEN), Hakodate College.

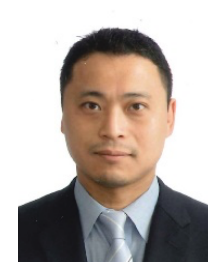

Kenji Moriya (Member) received Ph.D. degree from Muroran Institute of Technology in 2001, and is presently Professor of National Institute of Technology (KOSEN), Hakodate College since 2016. He has worked on biomeasurement engineering and signal processing. $\mathrm{He}$ is a member of IIAE, BMFSA, JSWE, JSEE and IEICE.

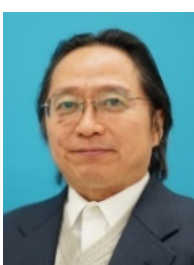

Masahiro Nakagawa (Non-member) received $\mathrm{Ph} . \mathrm{D}$. degree from Nagoya University in 1988, and is presently Professor of the department of science of technology innovation and the director of information processing center at Nagaoka University of Technology. His major discipline is concerned with non-linear phenomena in physical and physiological systems, e.g. chaos, fractal and neural networks, related to the brain function analysis focusing on the state-of-the-art in sensibility information engineering field. $\mathrm{He}$ is also a member of JPS, JSAP, JNNS, JLCS and IEICE et al. 